Military Technical College Kobry El-Kobbah, Cairo, Egypt

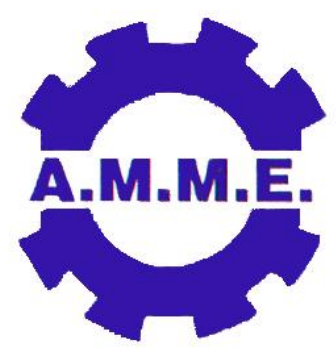

$14^{\text {th }}$ International Conference on Applied Mechanics and Mechanical Engineering.

\title{
Dynamic Analysis of Slider Crank mechanism and Two-Link Manipulator Using Constraint Technique
}

By

\author{
A. M. El-Nady ${ }^{\text {a }}$ Moutaz M. Hegaze ${ }^{\text {b }}$ and E. I. Imam Morgan ${ }^{c}$
}

\section{Abstract:}

The important of the slider crank mechanism and two-link mechanism is that they are the key-player in many mechanical and structural systems. A family of joints in form of library to carry out kinematical and dynamical analyses of mechanical systems was introduced in earlier work by authors [1]. Joint programming package was designed employing the proposed joint library. In this paper, parametric investigations have been carried out on two different types of mechanisms, closed loop mechanism (slider-crank mechanism) and open loop mechanism (two-link manipulator) to illustrate their effects on the response of the systems. The proposed applications have been described based on the type of joints and the number of degrees of freedom of the mechanism. Based on Lagrange multipliers theorem, the dynamic and inverse dynamic analyses have been carried out to calculate the reaction forces.

\section{Keywords:}

Constraint Technique, Slider Crank mechanisms, two-link mechanism

a Military Technical College, M. Sc., Mechanical Engineering Department b Military Technical College, Dr., Mechanical Engineering Department, corresponding author c German University in Cairo, Prof. Dr., Head of Mechatronics Department 


\section{Introduction}

Tremendous efforts in recent years were dedicated to the development of automated methods and to increase the degree of intelligence in computer-aided design (CAD) software. It is based on a computational method for studying the kinematics and dynamics of mechanical systems as first used multi-body dynamics formulations by Haug [2] and were implemented into commercial code DADS. Design parameters are modified to improve the performance. Based on experience and analytical formulations, a design may undergo a number of iterations before reaching an optimum situation [3]. This type of software facilitates constraint-based modeling and in many cases provides inference via constraint propagation. Some methods specify a parametric dependency between different parts. A design change in the geometry of a part propagates through the complete design. Other efforts at CAD/CAM automation include reasoning schemes that use topological relationships between features by McMahon [4]. More recent efforts to automate the mechanical design process in the manufacturing field were demonstrated by Abdel-Malek and Maropis [5]. The work based on recent rigorous mathematical formulations, was extended to the automatic computer aided design idea in Zou [6]. Jih-Lian $\mathrm{Ha}$ et al. [7] derived the equations of motion of a slider crank mechanisms using Hmilton's principle and Lagrange multiplier.

\section{Governing Equations in Planar Kinematics}

A body "i" can be located by specifying a body reference frame xi-yi which oriented by an angle $\theta i$ with respect to a global $x-y$ frame. Hence, the generalized coordinate vector of body "i" may be defined as,

$$
q_{i}=\left[\begin{array}{lll}
x_{i} & y_{i} & \theta_{i}
\end{array}\right]^{T}
$$

Which can be represented in terms of translation vector $u$ and rotation vector $\theta$ as follows:

$$
q_{i}=\left[\begin{array}{ll}
u & \theta
\end{array}\right]^{T}
$$

For a general planar mechanism of $\mathrm{N}$ rigid bodies, the number of planar generalized coordinates is $\mathrm{Nq}=3 \times \mathrm{N}$. The system generalized coordinate vector can be defined as:

$$
q=\left[\begin{array}{lllll}
q_{1}^{T} & q_{2}^{T} & q_{3}^{T} & \ldots & q_{N}^{T}
\end{array}\right]^{T}
$$

The governing equations in the motion of the planar mechanisms are the constraint equations between each two bodies

which impose conditions on the relative motion between them. When these conditions are expressed as algebraic equations in terms of generalized coordinates, they are called holonomic kinematic constraint $\mathrm{Nh}$ can be expressed in vector form as:-

$$
Q^{k}(q)=\left[Q_{1}^{k}(q), \ldots \ldots \ldots . . Q_{N_{h}}^{k}(q)\right]^{T}=0
$$


Hence, the system has NDOF independent coordinates which are called degrees of freedom, where

$$
\mathrm{NDOF}=\mathrm{Nq}-\mathrm{Nh}
$$

To analyze the motion of the mechanical system we must define an additional NDOF driving constraint that uniquely determines $\mathrm{q}(\mathrm{t})$ algebraically.

$$
Q^{D}(q, t)=0
$$

The kinematics and driving constraint equations $(4,6)$ can be combined in vector form as follows:

$$
Q(q, t)=\left[\begin{array}{ll}
Q^{K}(q, t) & Q^{D}(q, t)
\end{array}\right]^{T}=0
$$

Such a system is called Kinematically driven system.

\subsection{VELOCITY AND ACCELERATION DERIVATIONS}

The chain rule of differentiation has been used to obtain the velocity and acceleration equations by taken the first and second derivatives of the constraint equations (7) with respect to time as follows:

$$
\begin{gathered}
Q_{q} \dot{q}+Q_{t}=0 \\
Q_{q} \ddot{q}+\left(Q_{q} \dot{q}\right)_{q} \dot{q}+2 Q_{q t} \dot{q}+Q_{t}=Q_{q} \ddot{q}+\gamma=0
\end{gathered}
$$

Where

$$
\gamma=\left(Q_{q} \dot{q}\right)_{q} \dot{q}+2 Q_{q t} \dot{q}+Q_{t}
$$

This can be partitioned according to the definition of the generalized coordinate $q$ in equation (2) as follows:

$$
\begin{aligned}
& {\left[\begin{array}{ll}
Q_{u u} & Q_{u \theta} \\
Q_{\theta t} & Q_{\theta \theta}
\end{array}\right]\left[\begin{array}{c}
\dot{u} \\
\dot{\theta}
\end{array}\right]+\left[\begin{array}{c}
Q_{u t} \\
Q_{t \theta}
\end{array}\right]=0} \\
& {\left[\begin{array}{ll}
Q_{u t} & Q_{u \theta} \\
Q_{\theta t} & Q_{\theta \theta}
\end{array}\right]\left[\begin{array}{l}
\ddot{u} \\
\ddot{\theta}
\end{array}\right]+\left[\begin{array}{c}
\gamma_{u} \\
\gamma_{\theta}
\end{array}\right]=0}
\end{aligned}
$$

Where $Q q$ and $Q t$ are defined as the partial derivative of $Q$ with $q$ and $t$ respectively. For nonsingular Jacobin matrix Qq، the velocity can be obtained numerically as:

$$
\begin{gathered}
\dot{q}=-Q_{q}^{-1} Q_{t} \\
\ddot{q}=-Q_{q}^{-1} \gamma
\end{gathered}
$$

Newton-Raphson method has been used to solve the kinematically driven equations at each instant of time.

\subsection{Joint Library}


The objective of the joint library is to define a set of famous joints through its algebraic constraint equations to represent its physical function. The motion of any body is constrained relative to the global frame "absolute constraint" or relative to other body reference frame "relative constraint" [1].

\subsection{Dynamic Analyses}

Based on energy approach, the reaction forces that act on bodies are derived from Lagrange multipliers theorem which guarantees the existence of a Lagrange multiplier vector $\lambda$ such that [7],

$$
M \ddot{q}+Q_{q}^{T} \lambda=F
$$

This can be partitioned as follows:

$$
\left[\begin{array}{ll}
M_{u u} & M_{u \theta} \\
M_{\theta u} & M_{\theta \theta}
\end{array}\right]\left[\begin{array}{l}
\ddot{u} \\
\ddot{\theta}
\end{array}\right]+\left[\begin{array}{cc}
Q_{u u}^{T} & Q_{\theta u}^{T} \\
Q_{u \theta}^{T} & Q_{\theta \theta}^{T}
\end{array}\right]\left\lceil\begin{array}{c}
\lambda_{u} \\
\lambda_{\theta}
\end{array}\right]=\left\lceil\begin{array}{c}
F_{u} \\
F_{\theta}
\end{array}\right]
$$

Equations $(12,16)$ can be rearranged in mixed differential-algebraic matrix form as:

$$
\left[\begin{array}{cccc}
M_{u u} & M_{u \theta} & Q_{u u}^{T} & Q_{a t}^{T} \\
M_{\theta t} & M_{\theta \theta} & Q_{u \theta}^{T} & Q_{\theta \theta}^{T} \\
Q_{u u} & Q_{u \theta} & 0 & 0 \\
Q_{\theta u} & Q_{\theta \theta} & 0 & 0
\end{array}\right]\left[\begin{array}{c}
\ddot{u} \\
\ddot{\theta} \\
\lambda_{u} \\
\lambda_{\theta}
\end{array}\right]=\left[\begin{array}{c}
F_{u} \\
F_{\theta} \\
\gamma_{u} \\
\gamma_{\theta}
\end{array}\right]
$$

From equations $(11,12)$, the velocity and acceleration of the translation coordinates $u$ can be represented as:

$$
\dot{u}=Q_{u u}^{-l}\left(-Q_{u}-Q_{u} \dot{\theta}\right) \quad \& \quad \ddot{u}=Q_{u u}^{-l}\left(-\gamma_{u}-Q_{u} \ddot{\theta}\right)
$$

The coefficient $\lambda$ in equation (16) can be obtained as:

$$
\left[\begin{array}{l}
\lambda_{u} \\
\lambda_{\theta}
\end{array}\right]=\left[\begin{array}{ll}
Q_{u u}^{T} & Q_{\theta t}^{T} \\
Q_{u \theta}^{T} & Q_{\theta \theta}^{T}
\end{array}\right]^{-1}\left[\left[\begin{array}{c}
F_{u} \\
F_{\theta}
\end{array}\right]-\left[\begin{array}{cc}
M_{u u} & M_{u \theta} \\
M_{u \theta} & M_{\theta \theta}
\end{array}\right]\left[\begin{array}{l}
\ddot{\theta} \\
\ddot{\theta}
\end{array}\right]\right.
$$

From acceleration equation into equation (16) to obtain differential equation in the coordinates $\theta$ as:

$$
M^{v} \ddot{\theta}=F^{\theta}
$$

Runge-Kutta fourth method has been used to solve equation (19) in terms of generalized coordinate $\theta$.

\section{Slider Crank Mechanism}

The slider-crank mechanism shown in Figure 1 can be modeled using the constrained body analysis and the constraint library of joints developed earlier by the authors [1]. The slider-crank mechanism can be modeled in many different ways, one is introduced here to demonstrate the perpetration and the analysis of the data required for kinematic, dynamic and inverse dynamic analyses of this mechanism. 


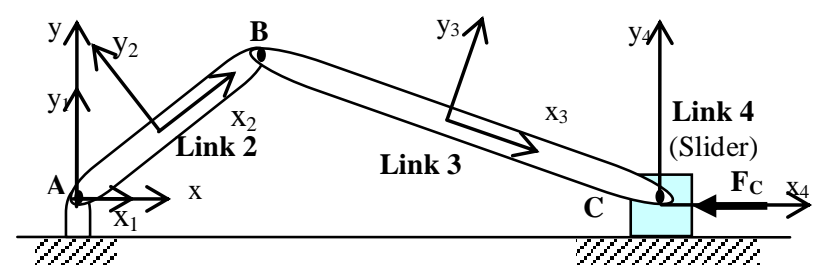

Figure 1. Slider-crank mechanism

The mechanism is modeled by 4 bodies, ground, crank, connecting rod and slider, with twelve generalized coordinates. Joints used herein are three revolute joints and absolute position constraints for the slider and ground which demonstrate eleven holonomic constraint equations. The data required for the joint modeling is illustrated in table 1.

Table. 1 data of the slider-crank joints

\begin{tabular}{ccccccccc}
\hline Joint & \multicolumn{2}{c}{ Revolute } & \multicolumn{2}{c}{ Revolute } & Revolute & Ground & Slider \\
\hline point & \multicolumn{2}{c}{$\mathrm{A}$} & \multicolumn{2}{c}{$\mathrm{B}$} & \multicolumn{2}{c}{$\mathrm{C}$} & $\mathrm{A}$ & $\mathrm{C}$ \\
\hline bodies & 1 & 2 & 2 & 3 & 3 & 4 & 1 & 4 \\
\hline $\mathrm{xi}$ & 0 & -1 & 1 & - & 0 & 0 & 0 & 0 \\
\hline $\mathrm{yi}$ & 0 & 0 & 0 & 0 & 0 & 0 & 0 & 0 \\
\hline$\varphi$ & & & & & & & 0 & 0 \\
\hline
\end{tabular}

It is clear that the mechanism has one degree of freedom, which must be specified to complete the modeling. If the crank rotates with angular velocity $2 \pi \mathrm{rad} / \mathrm{s}$ and driven constraint $\theta_{2}(t)=\frac{\pi}{4}+\omega t$, Table 2. Shows the geometric properties of the mechanism [2]:

Table 2. Properties of the slider-crank mechanism

\begin{tabular}{ccccc}
\hline Body Number & $\mathbf{1}$ & $\mathbf{2}$ & $\mathbf{3}$ & $\mathbf{4}$ \\
\hline Mass [kg] & 1 & 200 & 35 & 25 \\
\hline $\begin{array}{c}\text { Moment of inertia }[\mathrm{kg} \\
\left.. \mathrm{m}^{2}\right]\end{array}$ & 1 & 450 & 35 & 0.02 \\
\hline
\end{tabular}

\subsection{Kinematic analysis}

By using this model, kinematic analysis of the slider-crank mechanism is carried out using our own technique with a Matlab code. Since the motion of the slider (body 4 ) is of greatest concern in industrial applications, the position $(x)$, velocity $(\dot{x})$, and acceleration $(\ddot{x})$ of the slider are plotted for many different variations.

Two constant driving angular velocities $\omega_{1}=2 \pi \mathrm{rad} / \mathrm{s}$ and $\omega_{2}=4 \pi \mathrm{rad} / \mathrm{s}$ are considered, Figure 2.a. shows the acceleration, for the two driving constraints. Figure 2.b. shows the corresponding published results by Haug [2]. 


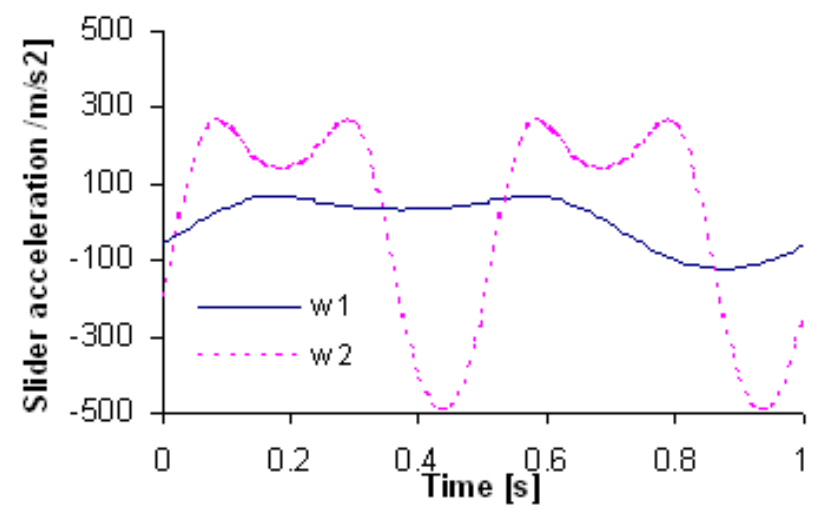

Figure 2.a. Time history of Slider acceleration with different angular velocities $(\omega)$

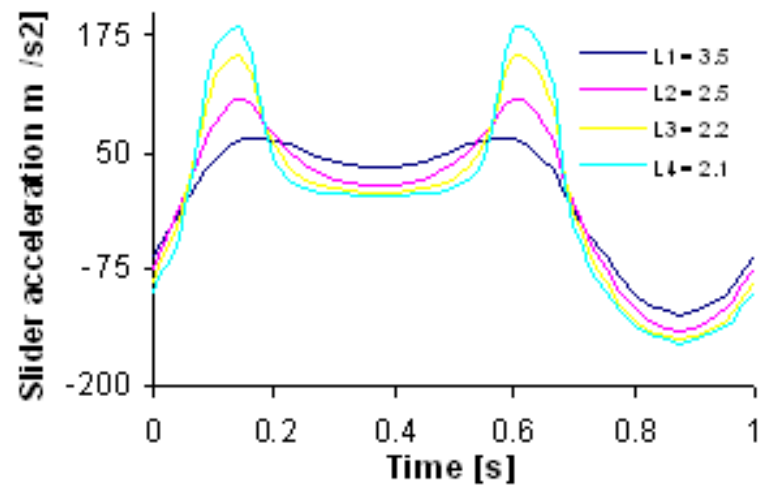

Figure 2.b. Time history of Slider acceleration with different connecting rod length

It is quiet convenient that the lock-up configuration will take place when the connecting rod (body 3) is shorter than the crank arm (body 2). Choosing the connecting rod length as $1.9 \mathrm{~m}$ which is shorter than the crank arm length $(L=2 \mathrm{~m})$. We observe that in Figure 3 . at time $t=$ $0.074 \mathrm{sec}$ both velocity and acceleration of the slider approach infinity, and the acceleration shows the more rapid divergence to infinity.

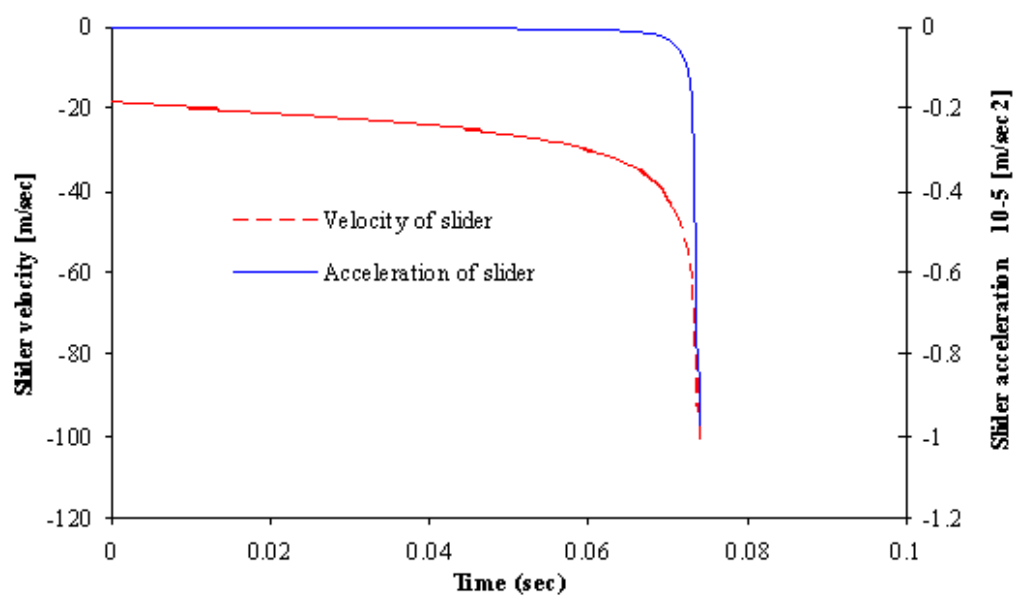

Figure 3. Velocity and acceleration of slider versus time (lock-up case)

\subsection{Inverse Dynamic}

The kinematic driving condition for the slider mechanism is used, to carry out inverse dynamic analysis. Gravitational force is neglected in this analysis. The torque required to achieve the constant angular displacement driving condition and the reaction force at the crank at the crank bearing ( joint A ) are calculated using Lagrange multipliers, as indicate earlier, and plotted in Figures 4 and 5 . respectively. 


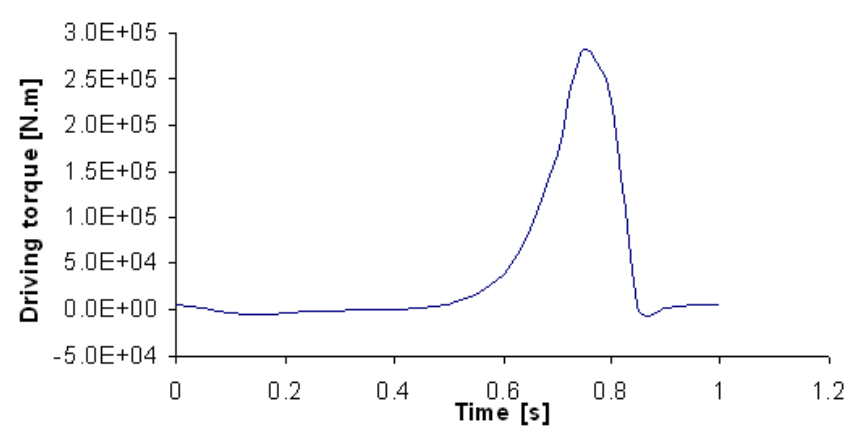

Figure 4. Driving torque, kinematically drevin Slider-crank

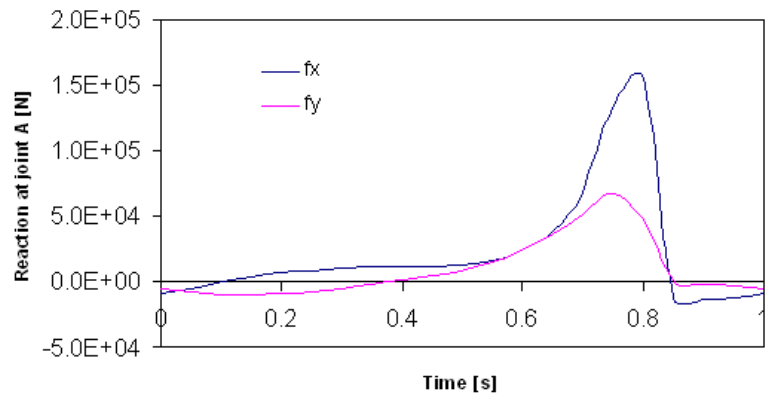

Figure 5. Reactions at joint $A$; kinematically drevin Slider-crank

\subsection{Dynamic analysis}

A torque of $14450 \mathrm{~N} . \mathrm{m}$ has been applied on the crank with initial angular velocity $30 \mathrm{rad} / \mathrm{s}$. Three simulations were carried out with different values of polar moment of inertia of the crank, ( $\mathrm{J} 2=225,450,900 \mathrm{~kg} . \mathrm{m} 2)$. As it is expected that, the larger the flywheel inertias will lead to less variation in the angular velocity of the fly wheel. Figures 6 and 7. confirm that behavior, both Figures show that, for the 1st choice of the inertia leads to the failure of the compressor to complete a single cycle.

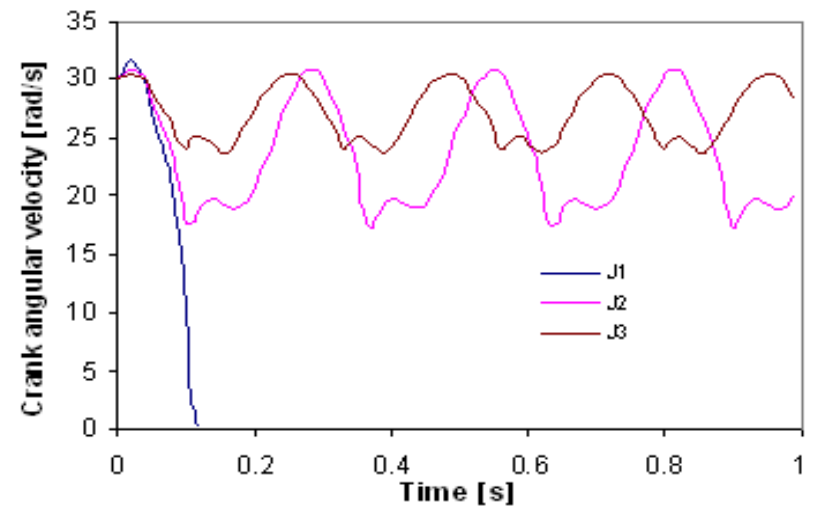

Figure 6. Angular velocity of crank with different flywheel inertias

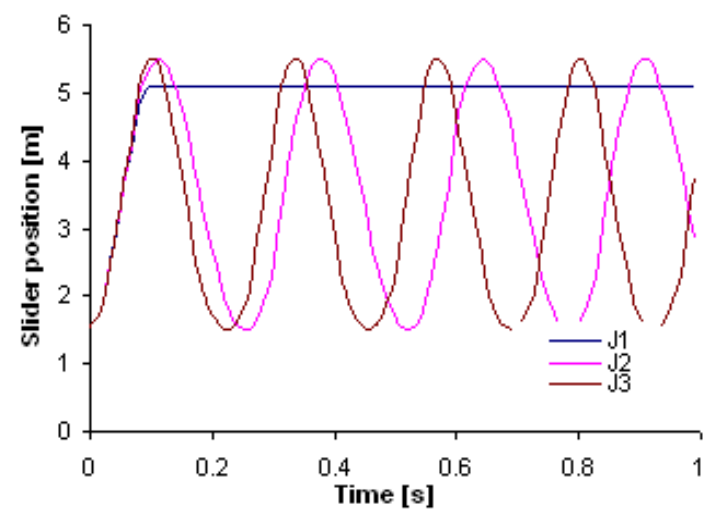

Figure 7. Time history of Slider position with different flywheel inertias

\section{Two-link mechanism}

The important of the two-link mechanism is that it is the key-player in many mechanical and structural systems. The geometry and inertia properties of the proposed model are shown in Figure 8. and table 3. 
Table 3. Properties of the two-link mechanism

\begin{tabular}{llll}
\hline Body Number & 1 & 2 & 3 \\
\hline Mass [kg] & 1 & 10 & 2 \\
\hline $\begin{array}{l}\text { Moment of inertia [kg } \\
\text {.m2] }\end{array}$ & $\begin{array}{l}10 \\
0\end{array}$ & 20 \\
\hline
\end{tabular}

Table 4. Data of the two-link mechanism

\begin{tabular}{|c|c|c|c|c|c|}
\hline Joint type & \multicolumn{2}{|c|}{$\begin{array}{c}\text { Revol } \\
\text { ute }\end{array}$} & \multicolumn{2}{|c|}{ Revolute } & $\begin{array}{c}\text { Grou } \\
\text { nd }\end{array}$ \\
\hline $\begin{array}{l}\text { Related } \\
\text { point }\end{array}$ & \multicolumn{2}{|c|}{ A } & \multicolumn{2}{|r|}{ B } & A \\
\hline $\begin{array}{c}\text { Related } \\
\text { bodies "i" }\end{array}$ & 1 & 2 & 2 & 3 & 1 \\
\hline$x_{i}$ & 0 & $\begin{array}{l}- \\
0 . \\
5\end{array}$ & $\begin{array}{l}0 . \\
5\end{array}$ & $0 . \overline{125}$ & 0 \\
\hline $\mathrm{y}_{\mathrm{i}}$ & 0 & 0 & 0 & 0 & 0 \\
\hline$\varphi$ & & & & & 0 \\
\hline
\end{tabular}

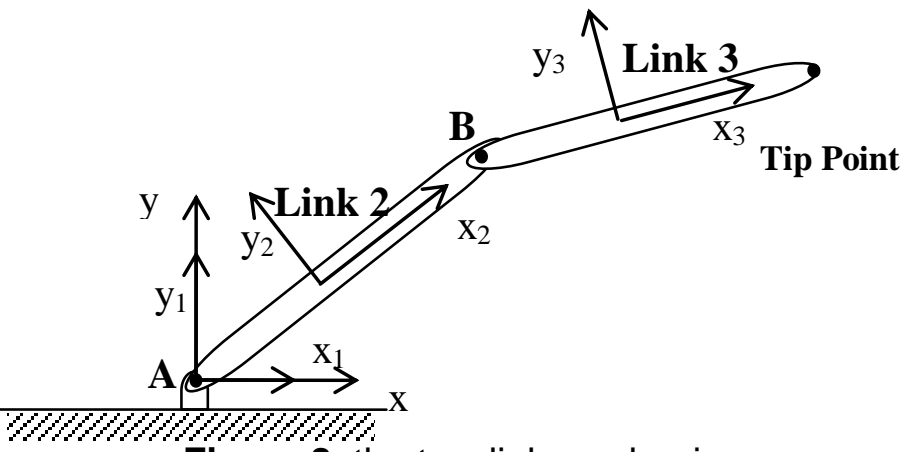

Figure 8. the two-link mechanism

The model has been analyzed using 3 bodies (ground and two links) and 3 joints (2 revolute joints and 1 ground) with the modeling data as shown in table 4, with nine generalized coordinates and seven holonomic constraint equations. It is quit clear that, the model has two degrees of freedom.

\subsection{Kinematical Analysis}

By using this model, kinematic analysis of the 2-Link mechanism is carried out using our own technique with a Matlab code, since the motion of the second link (body 3 ) in Figure 8 . is of greatest concern in manipulator design, the position, velocity, and acceleration of this link are plotted for many different variations in the driving constraints; these constraints can be represented graphically and analytically as shown in Figure 9 With $\mathrm{k}$ as a constant value (equals to 18), and the time period $\tau$ is chosen to be one sec.

$$
\phi^{1}=\left\{\begin{array}{cc}
\frac{k t^{2}}{2} & 0 \leq t<\frac{\tau}{2} \\
\frac{3}{4} k \tau^{2}-\frac{k}{2} t^{2}-k \tau . t & \frac{\tau}{2} \leq t<\tau \\
\frac{k}{4} \tau^{2} & \tau \leq t
\end{array} \quad \phi^{2}=\left\{\begin{array}{cc}
\frac{2 k}{3 \tau} t^{3} & \leq t<\frac{\tau}{4} \\
\frac{k \tau^{2}}{48}-\frac{k \tau \cdot t}{4}+k t^{2}-\frac{2 k t^{3}}{3 \tau} & \frac{\tau}{4} \leq t<\frac{\tau}{2} \\
\frac{k \tau^{2}}{48}-\frac{k \tau \cdot t}{4}+k t^{2}-\frac{2 k t^{3}}{3 \tau} & \frac{\tau}{2} \leq t<\frac{3 \tau}{4} \\
-\frac{13 k \tau^{2}}{24}+2 k \tau \cdot t-2 k t^{2}+\frac{2 k t^{3}}{3 \tau} & \frac{3 \tau}{4} \leq t<\tau \\
\frac{k \tau^{2}}{8} & \tau \leq t
\end{array}\right.\right.
$$




$$
\phi^{3}=\left\{\begin{array}{cc}
\frac{k}{\tau} t^{3} & 0 \leq t<\frac{\tau}{6} \\
\frac{k \tau^{2}}{216}-\frac{k \tau . t}{12}+\frac{k t^{2}}{2} & \frac{\tau}{6} \leq t<\frac{\tau}{3} \\
\frac{k \tau^{2}}{24}-\frac{5 k \tau \cdot t}{12}+\frac{3 k t^{2}}{2}-\frac{k t^{3}}{2} & \frac{\tau}{3} \leq t<\frac{\tau}{2} \\
\frac{k \tau^{2}}{24}-\frac{5 k \tau . t}{12}+\frac{3 k t^{2}}{2}-\frac{k t^{3}}{2} & \frac{\tau}{2} \leq t<\frac{2 \tau}{3} \\
-\frac{55 k \tau^{2}}{54}+\frac{11 k \tau . t}{12}-\frac{k t^{2}}{2} & \frac{2 \tau}{3} \leq t<\frac{5 \tau}{6} \\
-\frac{5 k \tau^{2}}{6}+3 k \tau \cdot t-3 k t^{2}+\frac{k t^{3}}{2} & \frac{5 \tau}{6} \leq t<\tau \\
\frac{k \tau^{2}}{6} & \tau \leq t
\end{array}\right.
$$

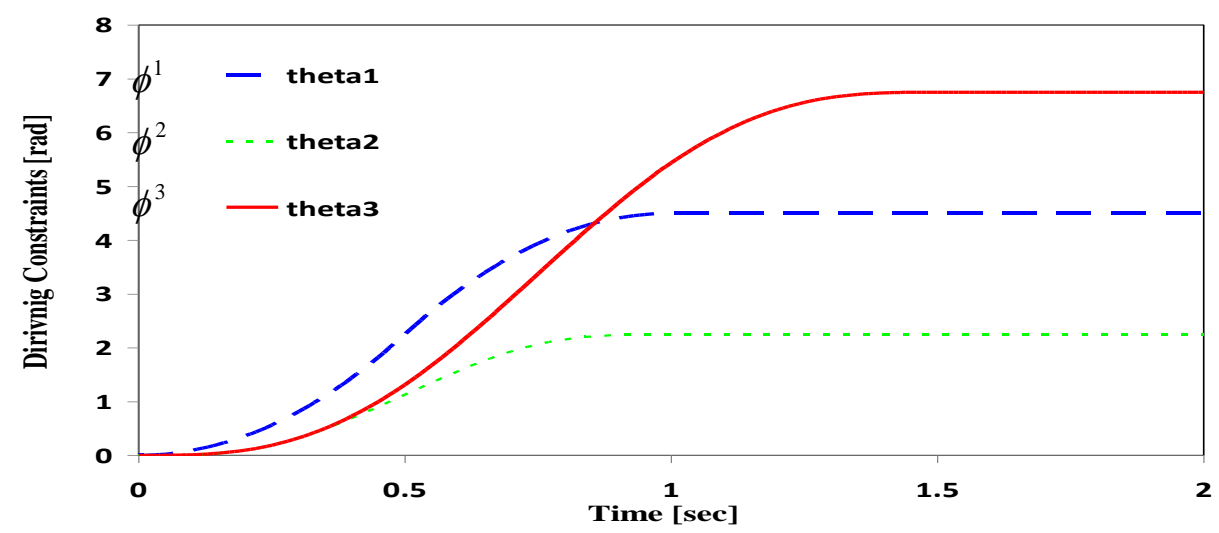

Figure 9. Different driving constraints represented graphically and analytically

The length of the first link (equals $1 \mathrm{~m}$ ) and the second is (equals $0.25 \mathrm{~m}$ ) links are held constant. Figures 10 and 11 show the horizontal and vertical positions of the tip with different driving constraints, respectively.

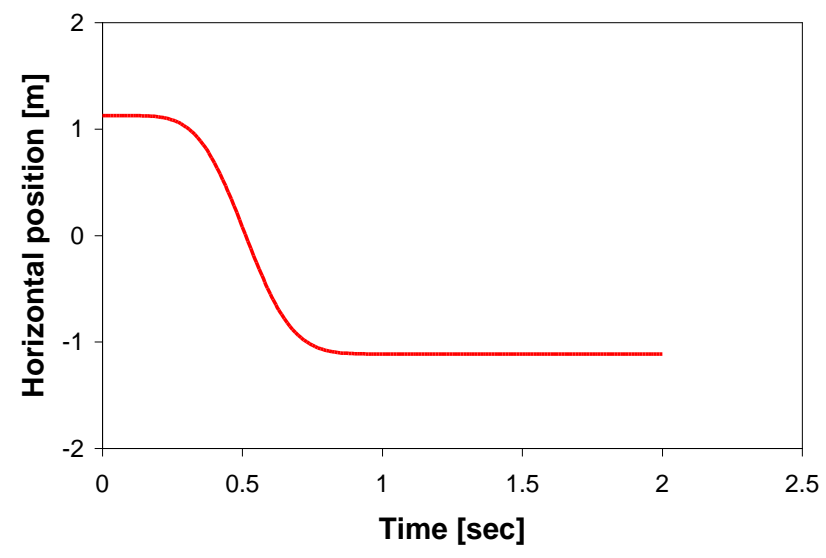

Figure 10. Time history of horizontal position of the Tip with $3^{\text {rd }}$ driving contraint

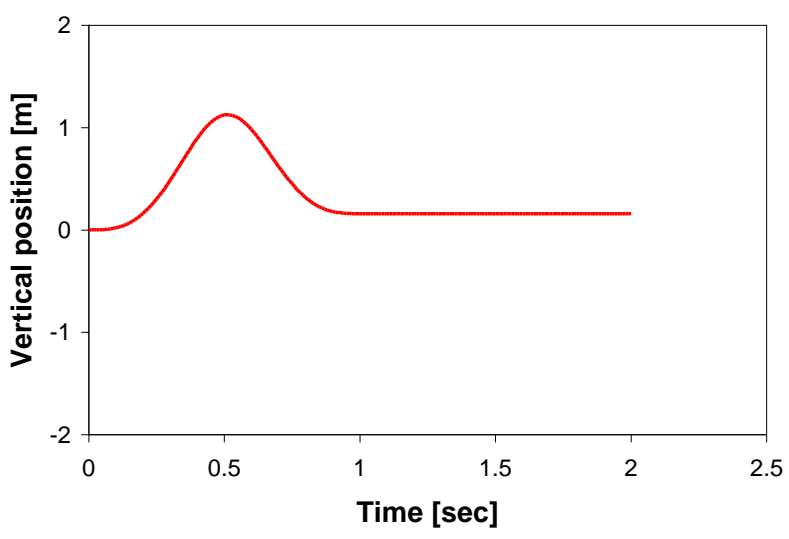

Figure 11. Time history of veritcal position of the Tip with $3^{\text {rd }}$ driving contraint

Figures 12 and 13 show the horizontal and vertical velocities of the tip with different driving constraints, respectively. 


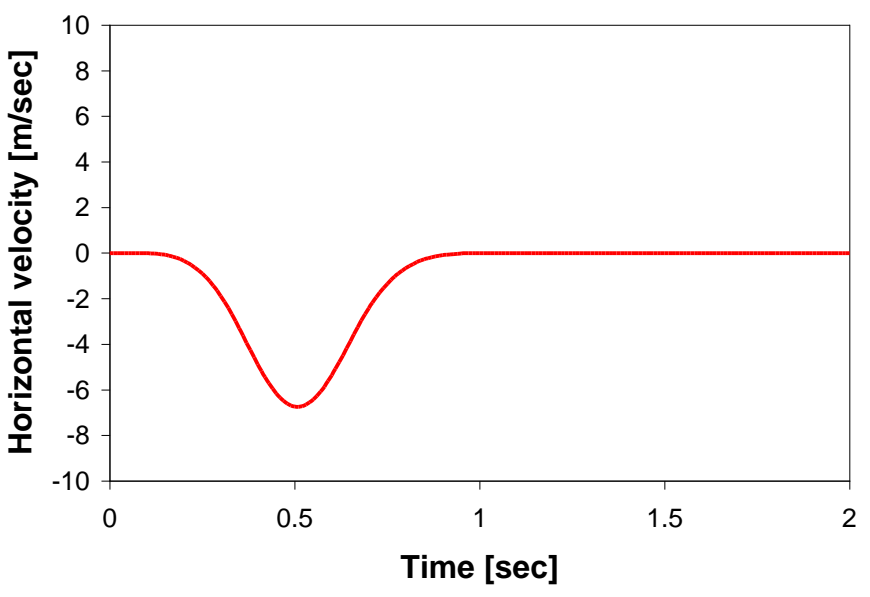

Figure 12. Time history of horizontal velocity of the Tip with $3^{\text {rd }}$ driving contraint

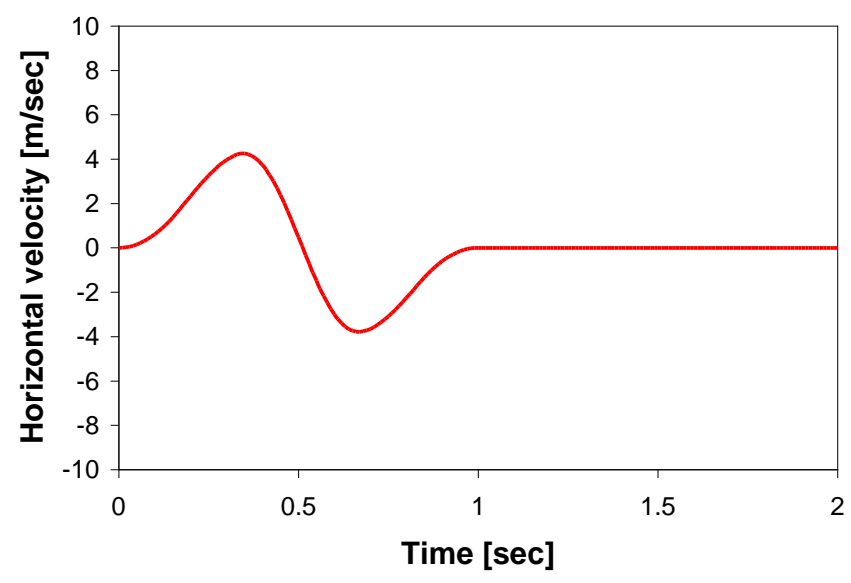

Figure 13. Time history of veritcal velocity of the Tip with $3^{\text {rd }}$ driving contraint

Figures 14 and 15 show the horizontal and vertical accelerations of the tip with different driving constraints, respectively.

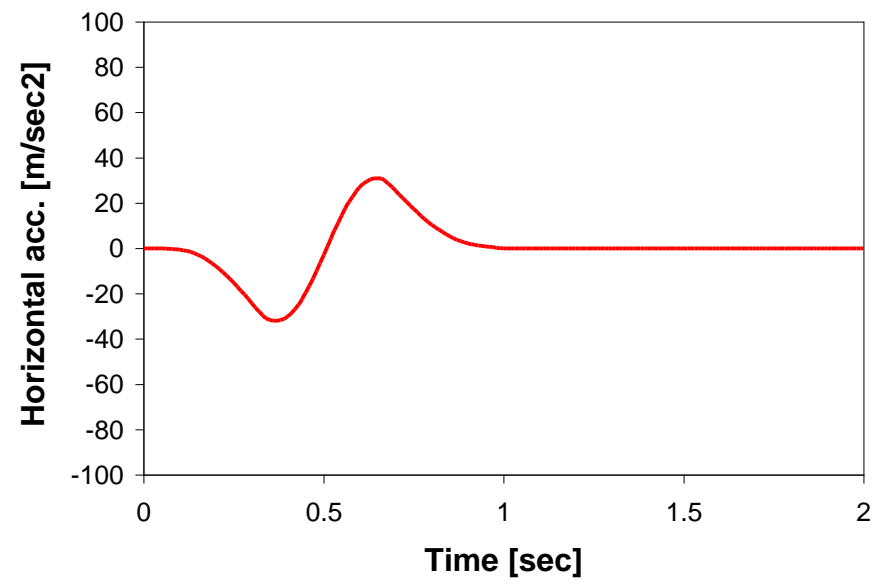

Figure 14. Time history of horizontal acceleration of the Tip with $3^{\text {rd }}$ driving contraint

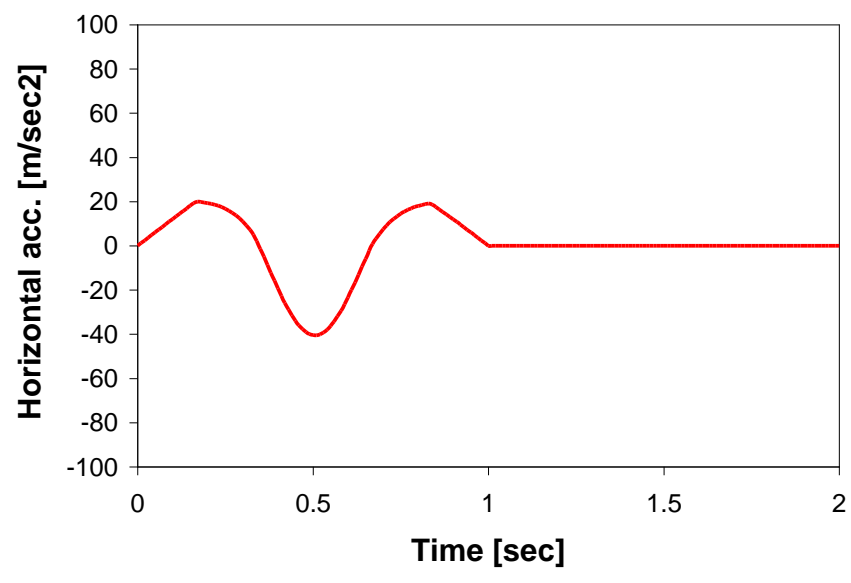

Figure 15. Time history of veritcal acceleration of the Tip with $3^{\text {rd }}$ driving contraint

A parametric study was carried out using different length of the second link, that is $L_{1}=0.25 \mathrm{~m}$, $L_{2}=0.5 \mathrm{~m}$, and $L_{3}=0.75 \mathrm{~m}$, the length of the first link is held constant and equals to $1 \mathrm{~m}$. We chose the third driving constraint as the operating driver. Figures 16 and 17 show the variation of the tip horizontal and vertical positions, respectively. Figures 18 and 19 show the variation of the tip horizontal and vertical velocity, respectively. Figures 20 and 21 show the variation of the tip horizontal and vertical velocity, respectively. 


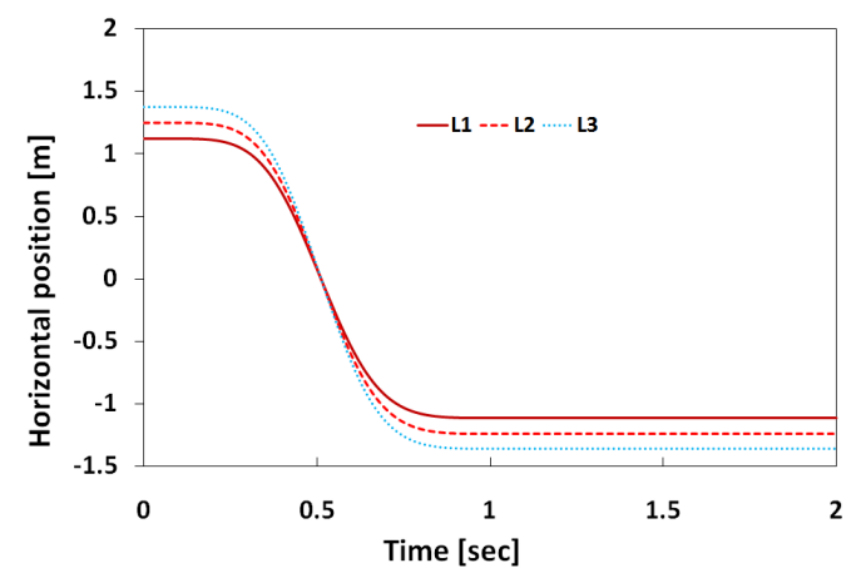

Figure 16. Time history of horizontal position of the Tip with different length of $2^{\text {nd }}$ link

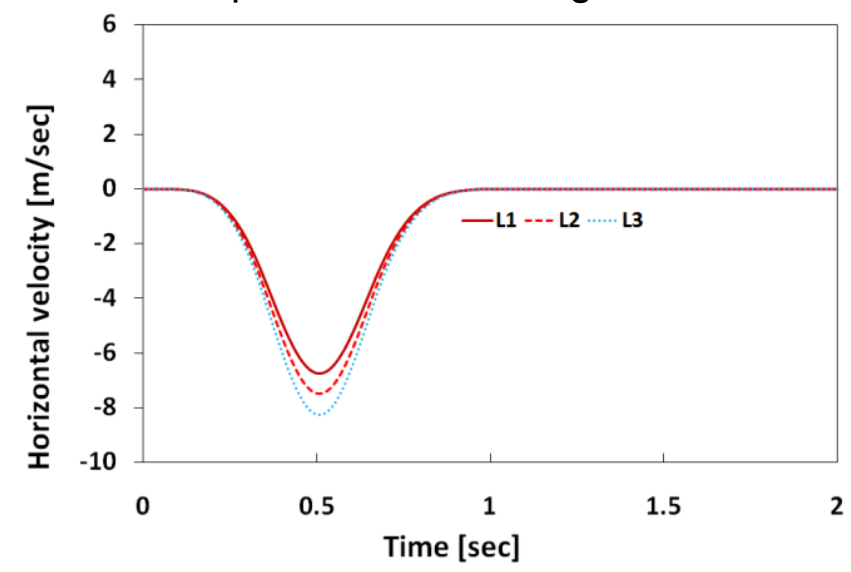

Figure 18. Time history of horizontal velocity of the Tip with different length of $2^{\text {nd }}$ link

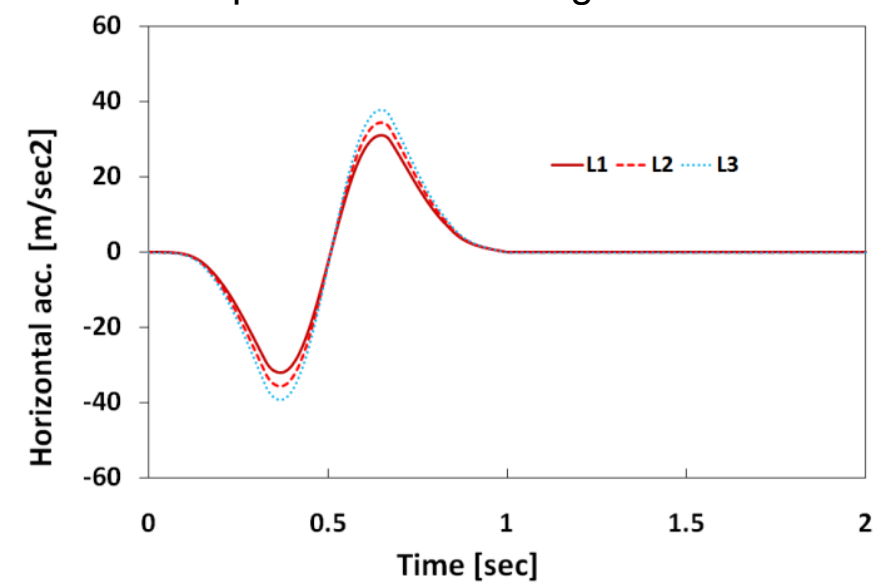

Figure 20. Time history of horizontal acceleration of the Tip with different length of $2^{\text {nd }}$ link

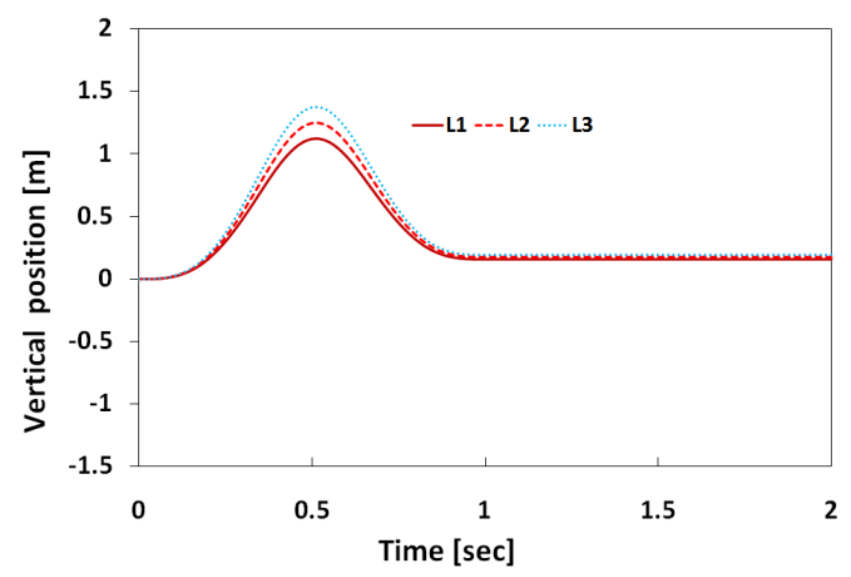

Figure 17. Time history of vertical position of the Tip with different length of $2^{\text {nd }}$ link

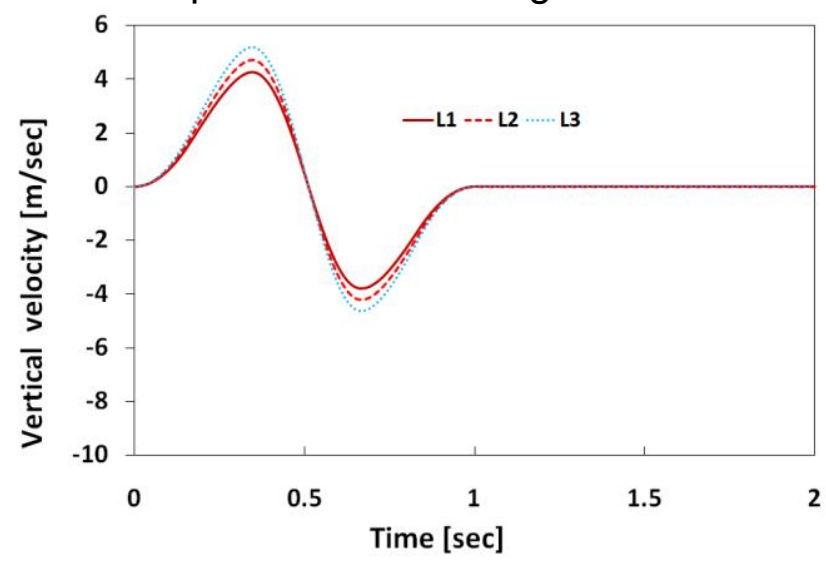

Figure 19. Time history of vertical velocity of the Tip with different length of $2^{\text {nd }}$ link

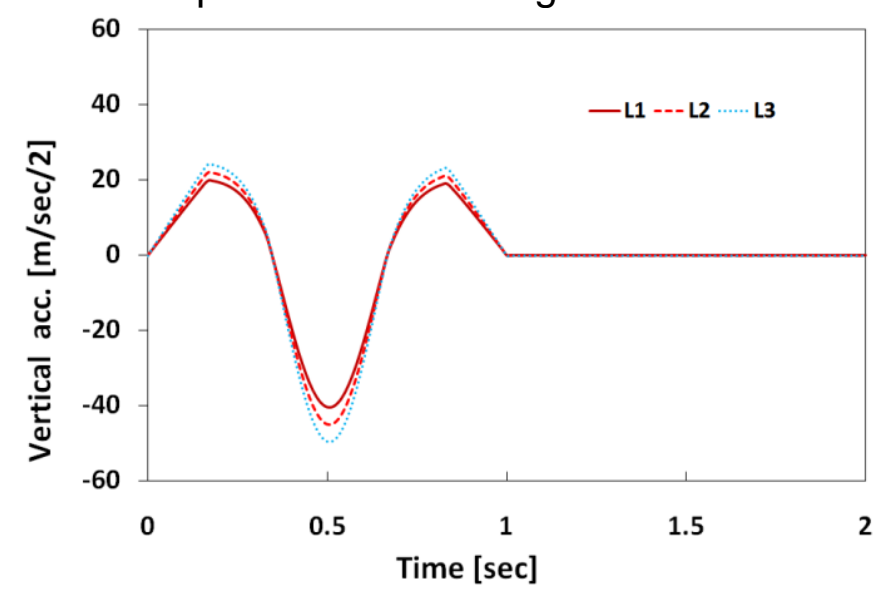

Figure 21. Time history of vertical acceleration of the Tip with different length of $2^{\text {nd }}$ link

\subsection{Inverse dynamic analysis}

The kinematic driving conditions prescribed in reference [1], to carry out inverse dynamic analysis. Gravitational force is neglected in this analysis. The torques required to achieve these angular driving conditions are calculated using Lagrange multipliers Figure 22 shows the driving 
torques for the 2 links of the mechanism that are required to achieve the third driving constraint $\phi^{3}$, Figures 23 and 24 show the reaction forces at joint A. Figures 25 and 26 show the reaction forces at joint $B$.

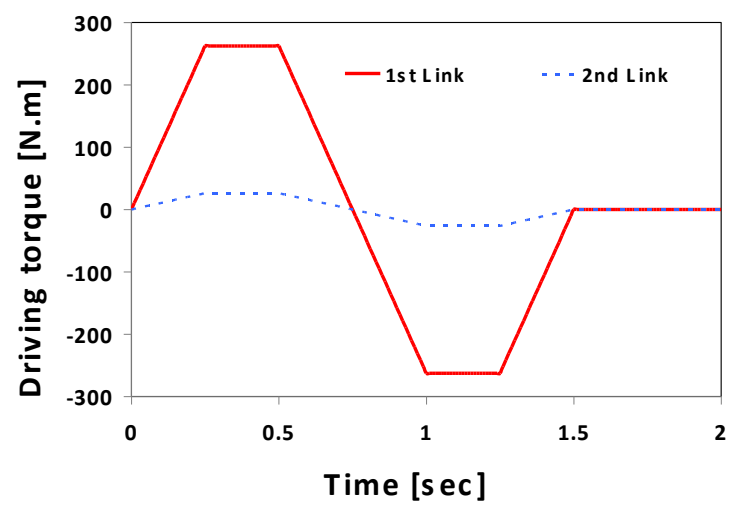

Figure 22. Driving torques of kinematically driven 2-Link mechansim

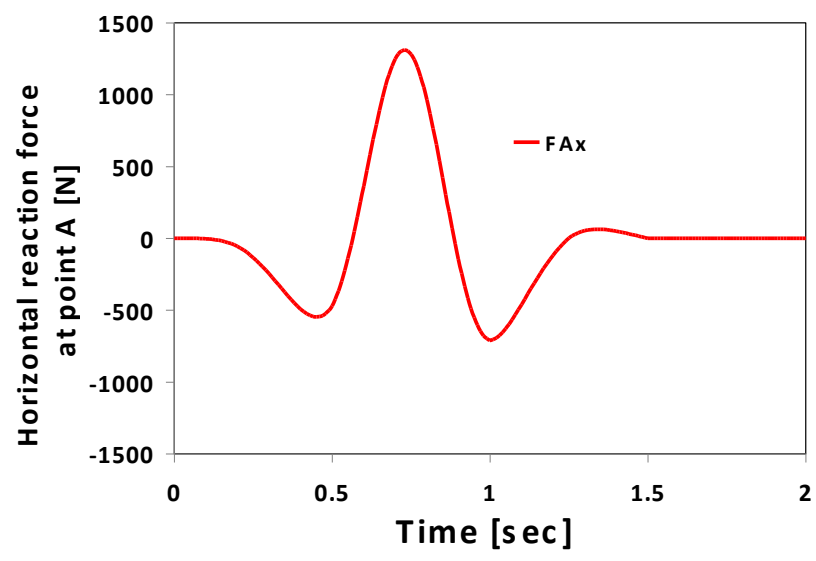

Figure 23. Time history of horizontal reaction force at joint $A$

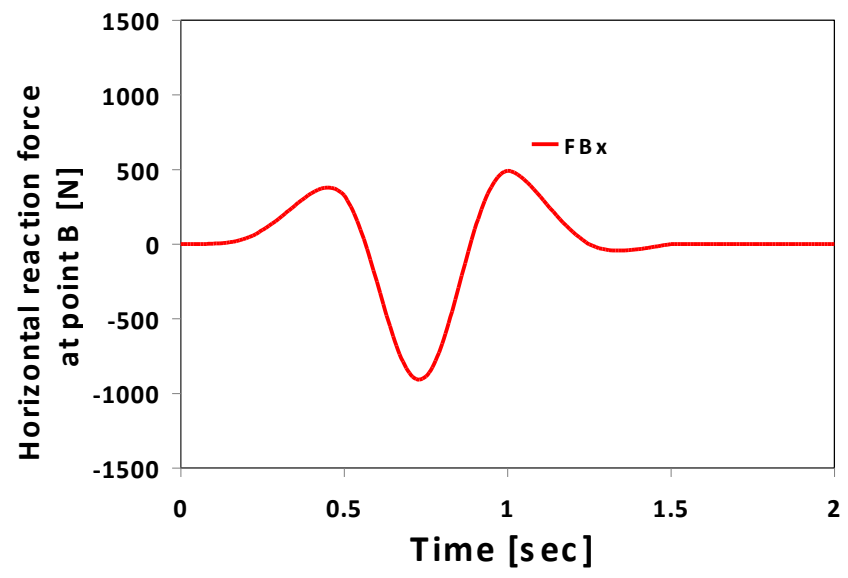

Figure 25. Time history of horizontal reaction force at joint $B$

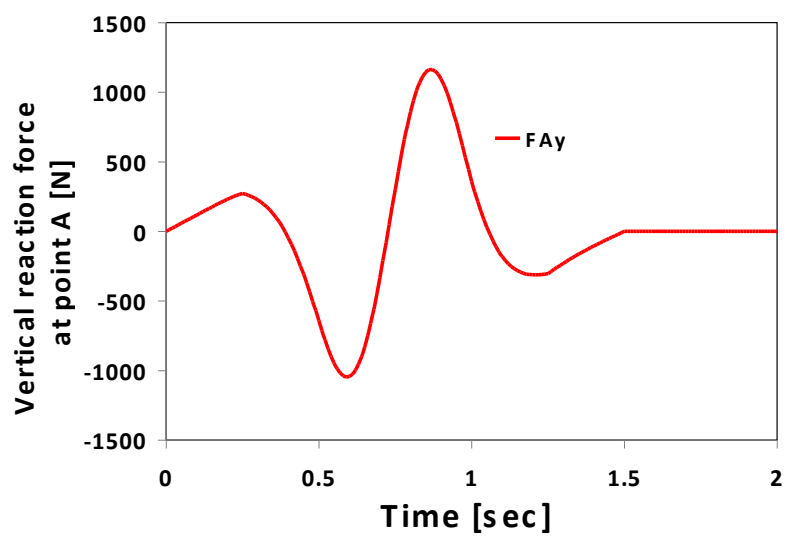

Figure 24. Time history of vertical reaction force at joint $A$

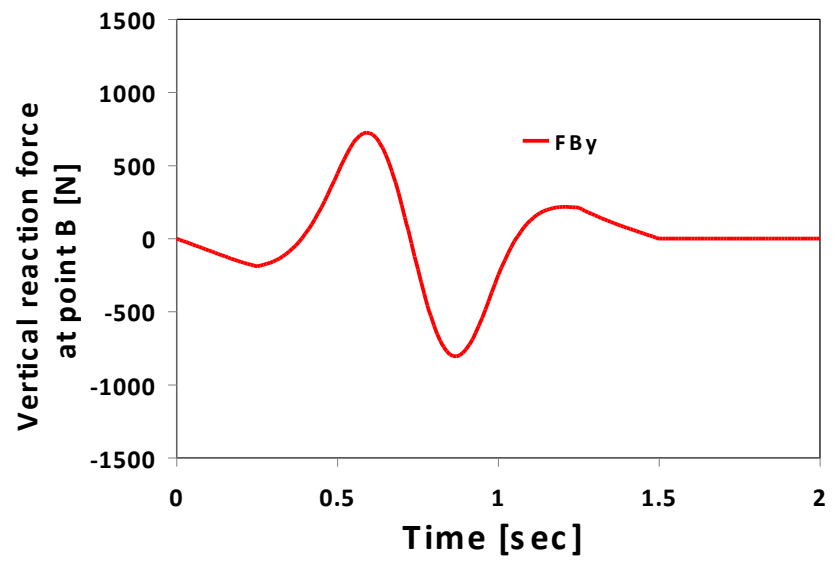

Figure 26. Time history of vertical reaction force at joint $B$ 


\subsection{Dynamic analysis}

In this section the 2-Link mechanism is analyzed with two different cases, in the first case the gravitational forces are neglected, and the input torques are equal to the torques resulted from the inverse dynamic analysis shown earlier in section 4.2 (similler to the step function torque) with no payload.

Motion begins at $t=0$, with initial conditions as follows:-

$$
\phi_{2}(0)=0 . \mathrm{rad}, \phi_{3}(0)=0 . \mathrm{rad} \dot{\phi}_{2}(0)=0 . \mathrm{rad} / \mathrm{sec}, \dot{\phi}_{3}(0)=0 . \mathrm{rad} / \mathrm{sec} .
$$

Where $\phi_{2}, \dot{\phi}_{2}$ are the angular position and velocity of the 1 st link, respectively and ' $\phi_{2}, \dot{\phi}_{2}$ are the angular position and velocity of the 2 nd link, respectively. A simulation was carried out with time period $\tau$ equals 2 seconds and $k=18$.

$$
T^{3}=\left\{\begin{array}{cl}
\frac{6 k}{\tau} t & 0 \leq t<\frac{\tau}{6} \\
k & \frac{\tau}{6} \leq t<\frac{\tau}{3} \\
\frac{6 k\left(\frac{\tau}{2}-t\right)}{\tau} & \frac{\tau}{3} \leq t<\frac{\tau}{2} \\
\frac{-6 k\left(t-\frac{\tau}{2}\right)}{\tau} & \frac{\tau}{2} \leq t<\frac{2 \tau}{3} \\
\frac{2 \tau}{3} \leq t<\frac{5 \tau}{6} \\
-\frac{6 k(\tau-t)}{\tau} & \frac{5 \tau}{6} \leq t<\tau \\
\tau & \leq t
\end{array}\right.
$$

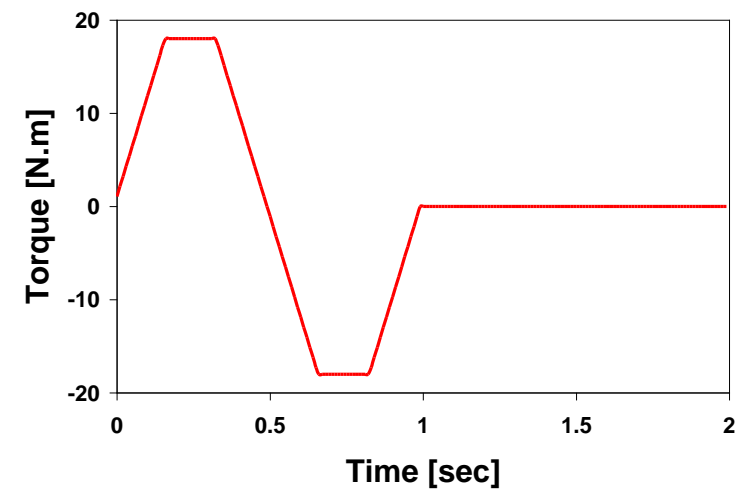

Figure 27. Analytical and graphical representation of the input Torque

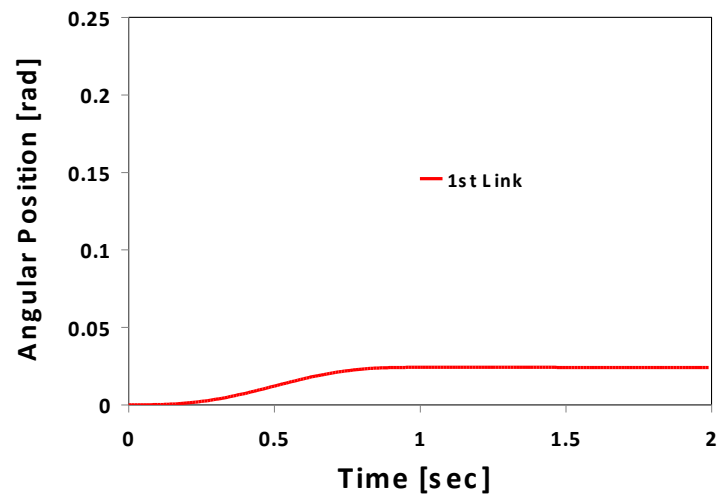

Figure 28. Time history of angular position of the $1^{\text {st }}$ link derived by input torque

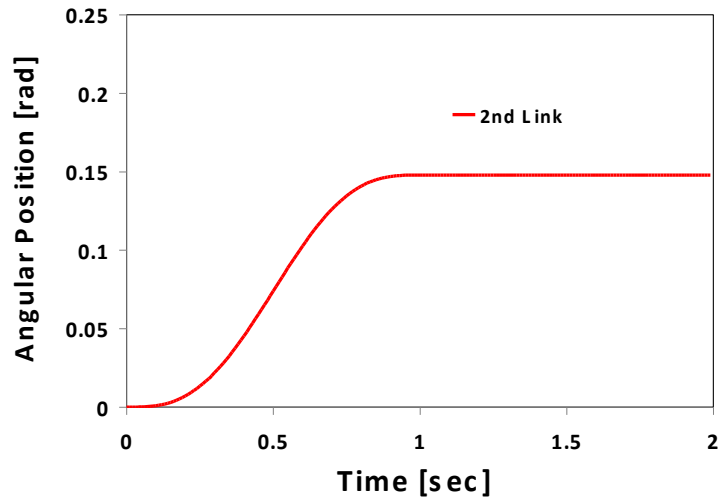

Figure 29. Time history of angular position of the $2^{\text {nd }}$ link derived by input torque 


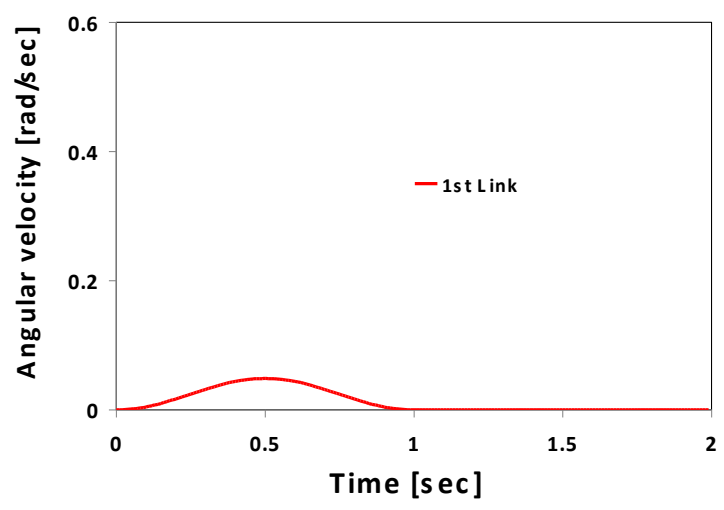

Figure 30. Time history of angular velocity of the $1^{\text {st }}$ link derived by input torque

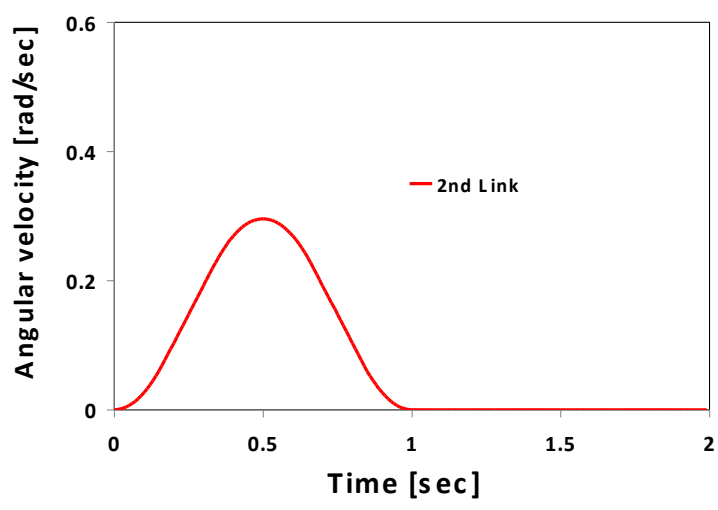

Figure 31. Time history of angular velocity of the $2^{\text {nd }}$ link derived by input torque

As it expected the resulted angular positions have the same nature of the input ones in the inverse dynamic analysis, which is carried out earlier. Figures 28 and 29. show the angular position of the two links with the input torque. Figures 30 and 31 . show the angular velocity of the two links.

\section{Conclusions}

The basic objectives of this work have been successfully achieved, and a powerful versatile computer package, which is capable of kinematic analysis and dynamic analysis for mechanical systems, have been developed based on advanced theory and efficient algorithms. The constraint equations of different types of joints have been introduced through joint library of two effective types of joints, revolute and prismatic joints. Kinematic and dynamic analyses of any mechanical system can be achieved via a run of the developed package, which manually considers the types of joints through an input data file by the user to carry out the required type of analysis. The dynamic analysis has been represented by mixed differential-algebraic equations of motion. The developed computer package has been successfully validated through comparison of its results with published results of slider-crank mechanism with different effective parameters (length of connecting rod). Parametric study has been carried out on slider-crank mechanism and 2-link manipulator.

\section{REFERENCES}

A. M. El-Nady, Moutaz M. Hegaze \& E. I. Imam Morgan "Application of Computational Mechanics Technique in Kinematic and Dynamic Analysis of Mechanical Systems Using Constraint Technique" Fourth International Conference on Advances and Trends in Engineering Materials and their Applications (AES - ATEMA' 2009 Hamburg)

Haug, E. J., 1989, Computer-Aided Kinematics and Dynamics of Mechanical Systems

Imamura, S, 1994, 'Development of constraint based object oriented language for engineering design on the parametric design support' Journal of the Japan Society for Precision Eng. Vol. 60 No 9, pp1242-1246

McMahon, C.A.; Cox, D.R.; Sims Williams, J.H.; Scott, J.A., 1997, "Representation and Reasoning in Computer Aided Process Planning," Proceedings of the Institution of Mechanical Engineers, Part B: Journal of Engineering Manufacture Vol. 211, pp 473-485. 
Abdel-Malek, K. and Maropis, N., 1998 "Design-to-Manufacture Case Study: Automatic Design of Post-Fabrication Mechanisms for Tubular Components," J.of Manufacturing Systems, Vol. 17, No. 3, pp. 183-195.

Zou, H.L. Abdel-Malek, K. and Wang, J., 1997a, "Design Propagation in Mechanical Systems: Kinematic Analysis," ASME Journal of Mechanical Design, Vol. 119, No. 3, pp. 338-345

Jih-Lian Haa, Rong-Fong Fungb,_, Kun-Yung Chenb, Shao-Chien Hsienb "Dynamic modeling and identification of a slider-crank mechanism" Journal of Sound and Vibration 289 (2006) 1019-1044 